\title{
Optimal Decoding and Performance Analysis of a Noisy Channel Network with Network Coding
}

\author{
Ming Xiao, Member, IEEE, and Tor Aulin, Fellow, IEEE
}

\begin{abstract}
We investigate sink decoding approaches and performance analysis for a network with intermediate node encoding (coded network). The network consists of statistically independent noisy channels. The sink bit error probability (BEP) is the performance measure. First, we investigate soft-decision decoding without statistical information on the upstream channels (the channels not directly connected to the sink). Numerical results show that the decoder cannot significantly improve the performance from a hard-decision decoder. We develop union bounds for analysis. The bounds show the asymptotic (regarding SNR: signal-to-noise ratio) performance of the decoder. Using statistical information about the upstream channels, we can find the error patterns of final hop channels (channels directly connected to sinks). With the error patterns, maximum-likelihood (ML) decoding can be performed, and a significant improvement in the BEP is obtained. To evaluate the union bound for the ML decoder, we use an equivalent point procedure. It is reduced to the least-squares problem with a linear constraint in the mediumto-high SNR region. With deterministic knowledge of the errors in the upstream channels, a genie-aided decoder can further improve the performance. We give the union bound for the genie decoder, which is straightforward to evaluate. By analyzing these decoders, we find that knowledge about the upstream channels is essential for good sink decoding.
\end{abstract}

Index Terms-Network coding, noisy channel, optimal decoding, bit error probability, transmitted energy and analysis bounds.

\section{INTRODUCTION}

$\mathbf{N}$ ETWORK coding was proposed in [1] to address the problem of network multicast capacity for applications in computer networks (e.g., Internet backbone). Thus, the problem of channel noise disturbance is assumed being solved ideally by powerful channel codes or retransmission in the physical layer. Then, it is natural to assume noiseless channels, i.e., error-free channels for network coding [1]. After [1], a lot of research has been done on network coding. In [2], linear coding is well addressed. It is shown in [2] that linear network coding is sufficient to achieve the min-cut capacity. In [3], an elegant algebraic framework is established for network coding. The network codes are well described using transfer matrices in [3]. Thus, the problem of finding an admissible network

Paper approved by O. Milenkovic, the Editor for Coding Theory and Applications of the IEEE Communications Society. Manuscript received May 4, 2007; revised October 19, 2007 and March 52008.

Part of this work was presented at the IEEE International Conference on Communications (ICC 2007), June 2007, Glasgow, UK.

M. Xiao is with the ACCESS Linnaeus Center, School of Electrical Engineering, Royal Institute of Technology, Sweden (e-mail: ming.xiao@ee.kth.se). He was with the Department of Computer Engineering, Chalmers University of Technology.

T. Aulin is with the Department of Computer Engineering, Chalmers University of Technology, Sweden (e-mail: aulin@chalmers.se).

Digital Object Identifier 10.1109/TCOMM.2009.05.070201 code is reduced to looking for nonsingular transfer matrices for the source-sink pairs.

In this paper, we will investigate the error probability of a coded network with noisy channels. Specifically, we will study the sink bit error probability (BEP) performance of various decoding approaches, and give analysis methods for them. The main motives are as follows: Firstly, for a communication network, especially for a system with finite processing capability or finite delay-tolerance, an error is inevitable due to channel noise. The separation of network and channel codes is asymptotical (regarding block length) [4], i.e., it needs an infinite block length and unlimited processing capability/delay [5]. This cannot be allowed in practice. A similar situation applies to point-to-point communications. It was shown that source and channel coding can be separated without loss of optimality when the block length goes to infinite [5], [6]. Yet joint source and channel coding has attracted lots of research [7], [8]. Further, in the intermediate node encoding and sink decoding process, noise disturbed transmitted symbols from different paths are mixed. Transmission errors might propagate in the network. Thus, the error probability is one of the essential performance measures for a coded network with noisy channels. Secondly, as shown later, decoding approaches have significant impact on the sink BEP of a coded network. How and why these decoders differ in performance have important impact on the network design. To the knowledge of the authors, these problems are mostly unexplored. Thirdly, though there are significant differences, some approaches used here are borrowed from point-to-point communications. Thus, it is valuable to investigate how the design and analysis approach of point-to-point channel codes can be used for network-correction codes.

The main contributions of this paper are as follows: We investigate the properties and performance of various sink decoding approaches. For the soft-decision decoder, we develop the union bound for analysis. Then, we know that though the decoder can improve the BEP performance relative to hard decision decoding, the improvement is not significant. This is due to the illegal final-hop codewords (to be defined). We propose an algorithm to collect the error patterns and their probabilities of final-hop codewords. The algorithm uses statistical information on the upstream channels. With error patterns and their probabilities, we can perform maximum-likelihood (ML) decoding. The decoder can significantly improve the BEP. We develop the union bound for the decoder, which uses an equivalent point procedure. It can be reduced to a least-squares problem with a linear constraint in the medium-to-high SNR (signal-to-noise ratio) region. If the decoders deterministically 
know the error pattern of the received codeword, the genieaided decoding approach can be used. It further improves the sink BEP. We show the union bound for the genie decoder, which is straightforward to evaluate. It serves as a performance lower bound for above decoders.

The organization of the paper is as follows. In Section II, we will introduce related work. In Section III, we describe the network model and define the performance measure. In Section IV, we analyze soft-decision decoding without statistical information about the upstream channels (the channels not directly connected to the sink). In Section V, we investigate ML decoding, which uses statistical information on the upstream channels. In Section VI, we discuss a genieaided decoder.

\section{RELATED WORKS}

Now we review literature related to network coding with noisy channels. Though the original work on network coding is for the error-free channels [1], network coding for a noisy channel network has attracted more and more research interest. In [9], the concept of network error correction is proposed and theoretical limits (Hamming bounds and GilbertVarshamov bounds) are derived. Reference [9] shows that network error correction is a generalization of point-to-point error correction. Since decoding approaches in point-to-point channel codes are essential for the performance [10], [11], optimal decoding in the sinks of a network using network coding (coded network) is also an important issue. Reference [4] shows that the channel and network codes can be separated without loss of optimality, if the codeword length of channel codes are infinitely long and the channels are statistically independent discrete memoryless channels (DMCs). Naturally, if these conditions are satisfied, information can be transmitted through every noisy channel in an error-free way by the Shannon theorem [5]. Thus, the problem of network coding for a network with noisy channels is reduced to network coding for networks with error-free channels, as in [1]. Yet, in [12], [13], network coding for networks with limited-processing in the intermediate nodes is considered. The papers investigate by mutual information the rate benefit of intermediate node encoding, and shows that channel and network coding usually cannot be separated without loss of optimality. A similar result is also shown in [14] for the deterministic networks without interference. Though references [12], [13] suggest that the sink should use ML decoding for optimality, how this decoding should be performed for a coded network is not given. In this sense, investigation of ML decoding for a coded network is a valuable topic. In [15], a lower bound on the alphabet size for the network correction code is addressed. Reference [15] discusses efficient encoding approaches of a coded network. Yet, an efficient decoding approach is not investigated. Packetlevel coding is considered for erasure channels in [16]. The erasure of packets might come from noise disturbance. In [17], streaming multicast with network coding is studied for wireless relay networks with noisy channels. Reference [17] shows that network coding can improve the throughput of noisy wireless networks with delay constraints. Due to space limitation, we do not list more papers on network coding with noisy channels. Readers are referred to [18] for more results on this issue.

\section{Network Model and Performance Measure}

The network here consists of one or more sources and sinks. We denote it as $G=<V, E>$, where $V$ and $E$ are the node set and the channel set, respectively. It is natural to describe the network with a directed graph as in [1], [2], [3]. The edges of the graph denote the channels. The channels are noisy and statistically independent. Then, the received observable (time-discrete vector) at the end of a channel is $\underline{r}=\underline{s}+\underline{n}$, where $\underline{s}$ is the transmitted signal vector of a single channel (including output channels of the source) in signal space, and $\underline{n}$ is the additive noise vector [19]. Note that matrices and vectors are underlined in this paper. If signal space has $l$-dimensions, $\underline{r}, \underline{s}$ and $\underline{n}$ can be geometrically denoted by points in $l$-dimensional Euclidean space $\mathbb{R}^{l}$. The signal can be, for example, multiple phase shift-keying (MPSK), pulse amplitude modulation (PAM) etc. Below, we will use binary phase shift-keying (BPSK) for illustration. Our principal results are more general, however. The signal space of BPSK is one dimensional $(l=1)$. The transmitted signal in channel $j$ is denoted as $\underline{s}_{j}$. We assume the channel noise to be additive white Gaussian noise (AWGN) [19]. Channel noise disturbs the transmitted signals, and this may cause a channel error, which occurs with a certain probability (channel error probability). Except for the sinks, the transmitted symbols are detected (hard-decision) at the end of each channel to form network code symbols. In the sink, the noise-disturbed BPSK signals are demodulated to form channel observations for the decoder. From $N$ input channels, the sink receives $N$ one-dimensional observations in each time slot. These $N$ observations form $N$-dimensional vectors $\underline{Y}$ and are described by points in $\mathbb{R}^{N}$. The decoder makes a decision from $\underline{Y}$. Clearly, $\underline{Y}$ corresponds to $N$ BPSK signals. These $N$ transmitted signals also form vectors (called transmitted vectors, and denoted as $\underline{X}$ ). Then, $\underline{Y}=\underline{X}+\underline{n}$, where $\underline{n}$ is an $N$-dimensional noise vector. Assuming these $N$ input channels to a sink are denoted as channel 1, channel $2, \cdots$, channel $N, \underline{X}=\left\{\underline{s}_{1}, \underline{s}_{2}, \cdots, \underline{s}_{N}\right\}$ is mapped from $N$ transmitted bits (denoted as $\underline{C}$ ). Note that $\underline{X}$ only denotes the vector of signals transmitted in the channels directly connected to the sinks. For convenience, $\underline{C}$ is called as a codeword. We use $E_{b}$ to denote the bit energy. For each bit of $\underline{C}, 1$ is mapped to $+E_{b}$, and 0 is to mapped to $-E_{b}$. We use a function $\pi(\cdot)$ to denote bit-wise mapping, i.e., $\underline{X}=\pi(\underline{C})$, denoting the BPSK signal vector of $\underline{C}$. $\underline{X}$ can also be described by points in $\mathbb{R}^{N}$. In [20], transmitted vectors in Euclidean space are called Euclidean space codes. Yet, the symbols of the codes in [20] are from different time slots. The symbols of $\underline{X}$ are for different channels. As we will show, there are many new properties of $\underline{X}$ from the intermediate node encoding, compared to [20]. If there are multiple sinks in a network, we assume that these sinks are separated in the topology (no direct channels between them). Thus, they cannot exchange decoding information. Each sink decodes using the channel observations only from its own input channels, i.e., there is no cooperation on decoding among different sinks. 
For the network, we make following assumptions: (1) The network is directed and acyclic. (2) To simplify the analysis, we assume binary network codes. Yet, the results here can be generalized to high alphabet-size codes. (3) The coding scheme is linear and deterministic. By deterministic coding, we mean that the coding scheme is fixed during the transmission process. (4) The network coding scheme is admissible, i.e., if there is no channel error, the sink can correctly reproduce the information bits.

To quantify the error probability performance, we use the BEP in the sink. It is defined as the probability in which any information bit reproduced in the concerned sink is erroneous. We call it the sink BEP (or briefly BEP) to distinguish it from the channel BEP. We assume that $w$ information bits are reproduced in the sink per time unit. An error event $c$ happens if $w_{c}\left(1 \leq w_{c} \leq w\right)$ bits of them differ from the correct one. This is a sink error event. If the probability of an error event is $P(c)$, the BEP of the event is $P_{b}(c)=P(c) \frac{w_{c}}{w}$, where $w_{c}$ the error weight of the event. Thus, the sink BEP can be evaluated by adding the BEPs of all error events [19], [11], i.e.,

$$
P_{b} \leq \sum_{c \in \varepsilon} P_{b}(c)=\sum_{c \in \varepsilon} P(c) \frac{w_{c}}{w},
$$

where $\varepsilon$ is the set of all error events. Equality holds only when error events are mutually exclusive (ME). Note that this definition of the BEP is for one specific sink. It slightly differs from the definitions of error probabilities in [14], [21], [22], where the BEP (or the probability of error events) is defined for the whole system. The motivation to use the sink BEP is that it denotes the quality of service $(\mathrm{QoS})$ between the sources and sinks, i.e., how reliable are the reproduced bits in the sinks? Do we have to improve the transmission approach? Also, the BEP measure attracts more and more research effort for Ad Hoc wireless networks [23], [24], [25]. This might be one of the most attractive applications for network coding [26], [27]. Here, the reproduced bit is the output from the sink decoder.

\section{Soft-Decision Decoding without Statistical INFORMATION ON UPSTREAM CHANNELS}

In this section, we shall study soft-decision decoding without statistical information on upstream channels. The statistical information means the channel error probability.

\section{A. System Description}

Without statistical information of upstream channels, the sink decoder only knows about the directly connected channels. This happens, for example, when the upstream channels are fast time-varying with unknown parameters, or there is no cooperation between the sink and them etc. Thus, the sink has to regard upstream channels as being error-free. It can only assume the correct codewords as candidates. The correct codeword is the codeword without transmission errors in upstream channels.

We use the example in Fig. 1 for illustration. Similar networks were shown in [12] to discuss the rate benefit of intermediate node encoding. Two bits $\left(b_{1}\right.$ and $\left.b_{2}\right)$ in the source $s$ are sent to the sink $t$. In the intermediate node $m_{3}$, two input

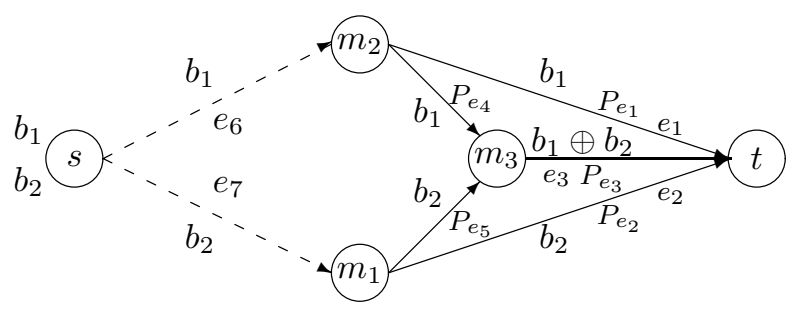

Fig. 1. A unicast with intermediate node encoding. The dashed lines $e_{6}$ and $e_{7}$ are error-free channels, and others are noisy channels.

bits are encoded (modulo- 2 addition) into one bit. Without loss of generality, we assume the channels $e_{1}, e_{2}, e_{3}, e_{4}$ and $e_{5}$ are disturbed by AWGN with a double side power spectral density (PSD) $N_{0} / 2$. The other two channels $\left(e_{6}, e_{7}\right)$ are error-free. The BEPs for $e_{4}$ and $e_{5}$ are $P_{e_{4}}$ and $P_{e_{5}}$, respectively. $P_{e_{4}}=$ $P_{e_{5}}=P_{e}=Q\left(\sqrt{2 \cdot E_{b} / N_{0}}\right)$, where the $Q(\cdot)$ function is the complementary Gaussian cumulative distribution function [19]. In the sink $t$, the received observations from the channels $e_{1}, e_{2}, e_{3}$ are denoted as $Y_{e_{1}}, Y_{e_{2}}$ and $Y_{e_{3}}$, respectively. The candidate codewords have the form $\underline{C}=\left\{b_{1}, b_{2}, b_{1} \oplus b_{2}\right\}$ for $e_{1}, e_{2}$ and $e_{3}$, respectively. The transmitted vector is denoted as $\underline{X}_{t}=\left\{X_{e_{1}}, X_{e_{2}}, X_{e_{3}}\right\}=\pi(\underline{C})$. The soft-decision decoder calculates the squared Euclidean distances between $\underline{Y}_{t}=\left\{Y_{e_{1}}, Y_{e_{2}}, Y_{e_{3}}\right\}$ and $\underline{X}_{t}$, i.e., $\sum_{i=1}^{3}\left(Y_{e_{i}}-X_{e_{i}}\right)^{2}$ for all candidate codewords. The final decisions for $b_{1}$ and $b_{2}$ are made for the minimum distance codeword [19], [11]. For convenience, we call the decoder a blind ML decoder, since the decisions are ML without upstream channel information.

To study performance, we simulate the network with the blind ML decoder. The output bits from the sink decoder are compared to source information $b_{1}$ and $b_{2}$. The number of error bits and transmitted bits are recorded. The simulation runs until the number of error bits reaches certain threshold (say 10,000). Fig. 2 shows the simulation results. In the figure, SNR $\left(E_{b} / N_{0}\right)$ in abscissa is for a single channel since we assume the same SNR for all channels. The BEP is the ratio of error bits in the sink $t$ to transmitted bits (both $b_{1}$ and $b_{2}$ )

In Fig. 2, we also show simulations of the hard-decision decoder, which uses a hard-decision ( 0 or 1$)$ from the demodulator. There are 4 matrices that can correctly reproduce the vector $\left\{b_{1}, b_{2}\right\}$ from $\underline{C}$ by right multiplication. They are $\left(\begin{array}{ll}1 & 0 \\ 0 & 1 \\ 0 & 0\end{array}\right),\left(\begin{array}{ll}0 & 0 \\ 1 & 1 \\ 1 & 0\end{array}\right),\left(\begin{array}{cc}1 & 1 \\ 0 & 0 \\ 0 & 1\end{array}\right)$, and $\left(\begin{array}{cc}0 & 1 \\ 1 & 0 \\ 1 & 1\end{array}\right)$. We assume that $P_{e} \ll 1$. To calculate the sink BEP with hard-decision decoding, we use the approach in [21], [22]. The approach enumerates the states of all channels in a network, and defines an error event occurring when one or more channels have transmission errors. For a network with independent channels, all ME error events and their probabilities can be collected in this way. Then, an algorithm using the modified transfer matrix is developed to find the error weight for each event. Thus, the sink BEP can be calculated. Using the approaches in [21], [22], we find the BEPs for these four decoding schemes to be approximately $P_{e}, 2.5 P_{e}, 4 P_{e}$ and $2.5 P_{e}$, respectively. The best decoder scheme has the sink BEP $P_{e}$. A better BEP 


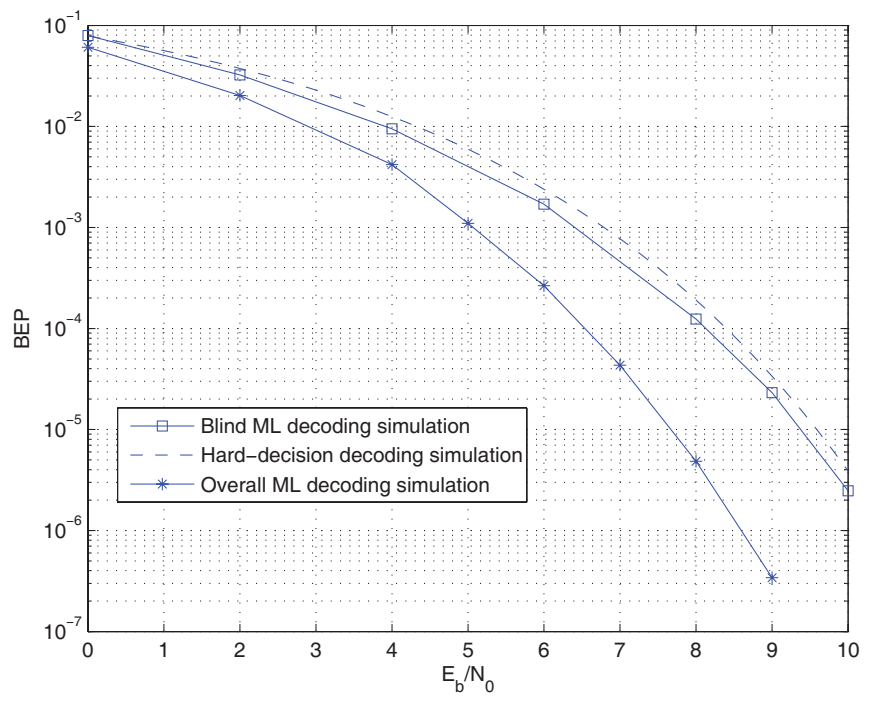

Fig. 2. Comparison of simulation results for the hard-decision decoding, soft-decision decoding and the overall ML decoding. Overall ML decoding will be discussed in Section V.

performance is obtained by soft-decision decoding as shown in simulations. Two curves are offset asymptotically (regarding SNR). Here, the asymptotic property is regarding the SNR except stated otherwise. The improvement is not significant. To disclose the decoder property and further improve performance, we analyze soft-decision decoding by union bounds.

\section{B. Bound Analysis}

To derive the union bound, we first make the following definitions:

Definition 1. Final hop node: The node directly connected to the sink by a channel. In Fig. $1, m_{1}, m_{2}$ and $m_{3}$ are the final hop nodes. All final hop nodes of a sink $t$ is called the final hop set of $t$. It is denoted as $\Phi(t)$. For the network in Fig. $1, \Phi(t)=\left\{m_{1}, m_{2}, m_{3}\right\}$.

Definition 2. Final hop channel: The channels directly connected to the sink. It is denoted as $E(t)$. We call $E(t)$ as the downstream channels of $\Phi(t)$. The upstream channel set is thus $\bar{E}(t)=E \backslash E(t)$.

Definition 3. Final hop codeword: The output bits (in the same time slot) transmitted from $\Phi(t)$ to the sink $t$. It is denoted $\underline{C}(t)$.

If the upstream channels are error-free, we can regard the final hop set as one super source node. All possible $\underline{C}(t)$ can be obtained by listing the source information bits. For example, in Fig. 1, if the channels $e_{4}, e_{5}, e_{6}$ and $e_{7}$ are error-free, the possible final hop codewords are $\{000,011,101,110\}$. We call the codewords under the error-free assumption for the upstream channels as the correct final hop codewords. The sink BEP performance can be analyzed using the traditional point-to-point approach if we regard the final hop set as the sender and the sink as the receiver.

However, this error-free assumption of the upstream channels is impractical for a multi-hop network. The final hop codeword cannot be collected by simply listing all source information bits. For example, in Fig. 1, $\{001\}$ is a possible codeword if channel $e_{5}$ or $e_{4}$ has one bit error. The error occurs with a certain probability. Without loss of generality, we assume all-zero bits as information bits. Then, the correct final hop codeword is also all-zero. Yet, due to transmission errors in the upstream channels, various final hop codewords can appear with certain probabilities. Thus, we must know all possible final hop codewords and their probabilities. For this, we make another definition:

Definition 4. Error pattern of the final hop codeword: The modulo- 2 result between a final hop codeword and the correct final hop codeword.

The error pattern is very important for optimal sink decoding. In addition to being used for bound analysis, it is essential to perform ML decoding (to be discussed). Since the final hop codewords appear with certain probabilities, the error patterns also occur with probabilities. It is only determined by the network topology, the coding scheme and channel BEPs, and is independent of the source information bits. This is the main reason for us to collect the error pattern instead of final hop codewords directly. For different source information, they share the same error patterns (for linear codes [11]). Thus, the problem of collecting final hop codewords is reduced to finding the error patterns. To collect error patterns, we enumerate the states of all upstream channels. Since the channels are independent, we can find all ME error events and their probabilities in the upstream channels. To find the error bits in the final hop set, we track how the channel errors propagate and cancel. This can be achieved using a modified transfer matrix. A detailed description is given in Appendix VII-A.

With the error patterns and their probabilities, we can obtain final hop codewords. Then, we can calculate the BEP by the traditional point-to-point approach since there is only one hop between the final hop set and the sink. We assume $J$ final hop codewords $\underline{C}_{1}, \underline{C}_{2}, \cdots, \underline{C}_{J}$ with probabilities $P\left(\underline{C}_{1}\right), P\left(\underline{C}_{2}\right), \cdots, P\left(\underline{C}_{J}\right)$. Since the error events causing the $J$ codewords are ME (Appendix VII-A), the probabilities of them add to 1 , i.e., $\sum_{i=1}^{J} P\left(\underline{C}_{i}\right)=1$. The BEP of softdecision decoding is:

$$
P_{b, s}=\sum_{i=1}^{J} P\left(\underline{C}_{i}\right) P_{b}\left(\underline{I} \mid \underline{C}_{i}\right),
$$

where $P_{b}\left(\underline{I} \mid \underline{C}_{i}\right)$ is the conditional BEP of soft-decision decoding when the final hop codeword $\underline{C}_{i}$ is transmitted. $\underline{I}$ denotes source information bits. For soft-decision decoding, $P_{b}\left(\underline{I} \mid \underline{C}_{i}\right)$ depends on the final hop channels and $\underline{C}_{i}$. Normally, the exact evaluation of $P_{b}\left(\underline{I} \mid \underline{C}_{i}\right)$ is quite difficult, and union bounds [19], [11] are used instead. Assuming $K$ candidate codewords, $P_{b}\left(\underline{I} \mid \underline{C}_{i}\right)$ is evaluated as:

$$
P_{b}\left(\underline{I} \mid \underline{C}_{i}\right) \leq \sum_{k=1}^{K} P\left(\underline{\widetilde{C}}_{k} \mid \underline{C}_{i}\right) \frac{w_{k}}{w}
$$

where $P\left(\widetilde{C_{k}} \mid \underline{C}_{i}\right)$ is the probability that the candidate codeword $\widetilde{C}_{k}$ is decoded (when the final hop codeword is $\underline{C}_{i}$ ), and $w_{k}$ is the error weight for $\widetilde{C}_{k}$. Equality holds only when the events behind $P\left(\widetilde{C}_{k} \mid \underline{C}_{i}\right)$ are ME. Each $\widetilde{C}_{k}$ corresponds to one $\underline{I}$ since only correct final hope codewords are assumed. Thus, we can get $w_{k}$ from $\underline{C}_{k}$. For instance, in Fig. 1, each $\widetilde{C_{k}}\left(\left\{b_{1}, b_{2}, b_{1} \oplus b_{2}\right\}\right)$ has one $\underline{I}\left(\left\{b_{1}, b_{2}\right\}\right)$. 


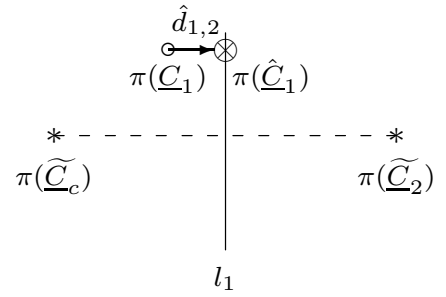

Fig. 3. Geometric representation for the transmitted vectors of candidate codewords and an illegal codeword in $\mathbb{R}^{2} . \widetilde{C}_{c}$ is the correct codeword and $\underline{C}_{1}$ is the transmitted final hop codeword.

In general, it is very difficult to evaluate $\operatorname{ME~} P\left(\widetilde{\widetilde{C}}_{k} \mid \underline{C}_{i}\right)$ s, since the integral on Gaussian distribution (from the distribution of AWGN) is quite difficult to evaluate ([11], [19]), especially when the decision regions of $\widetilde{C_{k}}$ in signal space are irregular. Thus, the conditioned pair-wise probabilities $P\left(\underline{\widetilde{C}}_{c} \rightarrow{\widetilde{C_{k}}}_{k} \mid \underline{C}_{i}\right)$ are used [19], [11]. It means the probability that $\widetilde{C}_{k}$ is decoded when only ${\underline{C_{k}}}_{k}$ and $\widetilde{C_{c}}$ are considered as the candidate codewords, conditioned that $\underline{C}_{i}$ is transmitted. $\underline{C}_{i}$ may be neither of them. Here $\widetilde{C}_{c}$ is the correct final hop codeword. Thus, there are only two decision regions in signal space. One is for $\widetilde{C}_{c}$ (denoted as $\hat{R}_{c}$ ) and another is for $\widetilde{C}_{k}$ (denoted as $\hat{R}_{k}$ ). The probability of $\widetilde{C_{k}}$ being decoded is the probability that noise moves $\underline{C}_{i}$ into $\hat{R}_{k}$. For AWGN channels, $P\left(\underline{\widetilde{C}}_{c} \rightarrow{\widetilde{C_{k}}}_{k} \mid \underline{C}_{i}\right)$ is [19]

$$
P\left(\underline{\widetilde{C}}_{c} \rightarrow \widetilde{\widetilde{C}}_{k} \mid \underline{C}_{i}\right)=Q\left(\hat{d}_{i, k} \sqrt{\frac{2}{N_{0}}}\right),
$$

where $\hat{d}_{i, k}$ is the vector distance between $\pi\left(\underline{C}_{i}\right)$ and its orthogonal projection (denoted as $\hat{C}_{i}$ ) on the border between $\hat{R}_{k}$ and $\hat{R}_{c}$. It is defined as $\hat{d}_{i, k}^{2} \triangleq(-1)^{\delta_{i, k}} \sum_{i=1}^{n}\left|X_{i}-\hat{X}_{i}\right|^{2}$, where $\pi\left(\underline{C}_{i}\right)=\left\{X_{1}, X_{2}, \ldots, X_{n}\right\}$ and $\pi\left(\hat{C}_{i}\right)=\left\{\hat{X}_{1}, \hat{X}_{2}, \ldots, \hat{X}_{n}\right\}$. $\delta_{i, k}=1$ if $\pi\left(\underline{C}_{i}\right)$ lies inside $\hat{R}_{k}$, otherwise $\delta_{i, k}=0$. Thus, $\hat{d}_{i, k}$ is negative if $\pi\left(\underline{C}_{i}\right)$ lies inside $R_{k}$. In this case, $P\left(\widetilde{C}_{c} \rightarrow \widetilde{C}_{k} \mid \underline{C}_{i}\right)$ means the probability in which $\pi\left(\underline{C}_{i}\right)$ is inside $\hat{R}_{k}$ and the channel noise moves it out (of $\hat{R}_{k}$ ). One example is shown in Fig. 3 for illustration. In a 2-dimension Euclidean space $\left(\mathbb{R}^{2}\right)$, there are two transmitted vectors of two candidate codewords $\widetilde{C}_{c}$ and $\widetilde{C}_{2} \cdot \underline{C}_{1}$ is the transmitted final hop codeword, and it is unknown to the sink (illegal codeword). The border of the decision regions for $\widetilde{C}_{c}$ and $\widetilde{C}_{2}$ is the solid line $l_{1}$. We shall calculate $P\left(\widetilde{C}_{c} \rightarrow \widetilde{C}_{2} \mid \underline{C}_{1}\right)$, the probability in which $\underline{C}_{1}$ is transmitted and $\underline{C}_{2}$ is decoded. It equals the probability in which noise moves $\pi\left(\underline{C}_{1}\right)$ to the decision region of $\widetilde{C}_{2}$. The projection of $\pi\left(\underline{C}_{1}\right)$ on $l_{1}$ is $\pi\left(\hat{C}_{1}\right)$. Thus, $P\left(\underline{\widetilde{C}}_{c} \rightarrow{\underline{C_{2}}}_{2} \mid \underline{C}_{1}\right)=Q\left(\hat{d}_{1,2} \sqrt{\frac{2}{N_{0}}}\right)$, where $\hat{d}_{1,2}=+\left|d_{1,2}\right| \cdot\left|d_{1,2}\right|$ is the Euclidean distance between $\pi\left(\underline{C}_{1}\right)$ and $\pi\left(\hat{C}_{1}\right)$. $\hat{d}_{1,2}$ is positive since $\pi\left(\underline{C}_{1}\right)$ lies outside of the decision of $\widetilde{C}_{2}$.

Hence, we can calculate the union bound for soft-decision decoding. The detailed calculation for the example in Fig. 1 is shown in Appendix VII-B. The results are shown in Fig. 4. By analyzing final hop codewords, we notice that the final hop codewords might not belong to any candidate codewords, due to the errors in the upstream channels. We

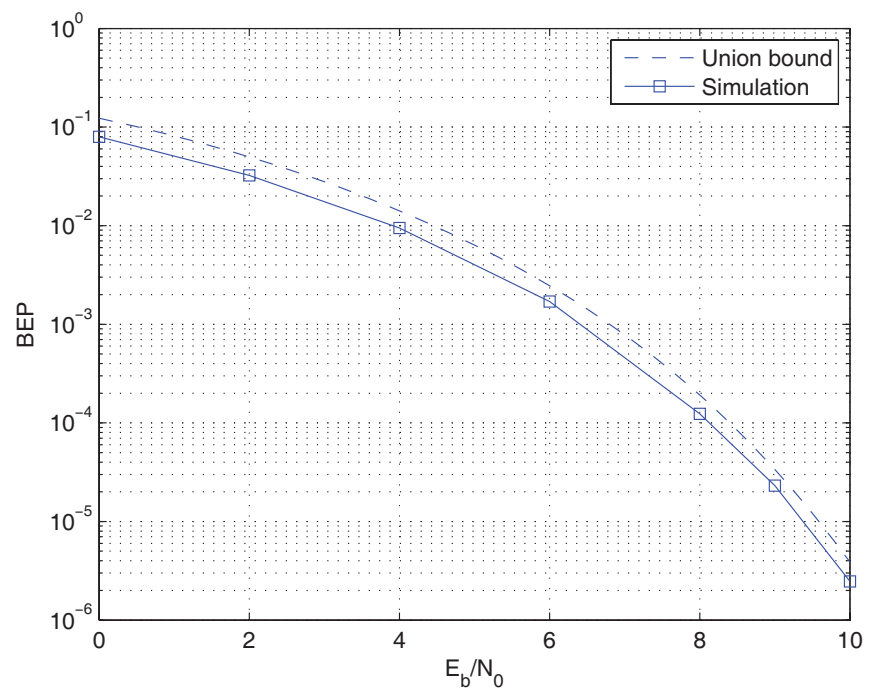

Fig. 4. The simulation results and union bound for soft-decision decoding.

call these codewords illegal codewords (e.g., $\{001\}$ in Fig. 1). They cannot be found out by the final hop nodes since they are separate. Illegal codewords are often devastating to sink performance, since they may lie in the border of decision regions of correct codewords and wrong codewords. A little noise can cause a decoding error (see, e.g., Appendix VII-B).

Proposition 1: The above union bound is either asymptotically tight or asymptotically offset.

Proof: The final hop codewords and their probabilities $P\left(\underline{C}_{i}\right)$ are exact results. We evaluate $P_{b}\left(\underline{I} \mid \underline{C}_{i}\right)$ using (3). If $\underline{C}_{i}$ is an illegal codeword and one or more $\hat{d}_{i, k} \leq 0\left(\hat{d}_{i, k}<0\right.$ when $\pi\left(\underline{C}_{i}\right)$ in the decision region of $\left.\widetilde{C}_{k}\right), P\left(\underline{\widetilde{C}}_{c} \rightarrow \underline{C}_{k} \mid \underline{C}_{i}\right)$ approaches a constant with increasing SNR and dominates $P_{b}\left(\underline{I} \mid \underline{C}_{i}\right)$. Then, $P_{b}\left(\underline{I} \mid \underline{C}_{i}\right)$ approaches a constant with increasing SNR. $P\left(\underline{C}_{i}\right)$ asymptotically dominates the bound. Several constant $P\left(\underline{C}_{c} \rightarrow \underline{C}_{k} \mid \underline{C}_{i}\right)$ s with overlapping decision regions up-shift the curve of $P\left(\underline{C}_{i}\right)$. If all $\hat{d}_{i, k}>0\left(\underline{C}_{i}\right.$ is a legal codeword), then $P\left(\widetilde{C}_{c} \rightarrow \underline{C}_{k} \mid \underline{C}_{i}\right)$ with the smallest distance will asymptotically dominate $P_{b}\left(\underline{I} \mid \underline{C}_{i}\right)$. The bound will be asymptotically tight. Q.E.D.

Thus, the BEP performance for the soft-decision decoding is well evaluated. We can explain why soft-decision decoding and hard-decision decoding are asymptotically offset. In Fig. 1, due to the illegal codeword $\underline{C}_{1}=\{001\}, P_{b, s}$ is asymptotically determined by $P\left(\underline{C}_{1}\right) \approx A_{1} P_{e_{4}}=A_{1} Q\left(\sqrt{\frac{2 E_{b}}{N_{0}}}\right)$. The asymptotical performance of the hard-decision decoding is $P_{b} \approx A_{2} P_{e_{1}}=A_{2} Q\left(\sqrt{\frac{2 E_{b}}{N_{0}}}\right)$. Here $A_{1}$ and $A_{2}$ are constants, and we assume $P_{e_{1}}=P_{e_{2}}$ and $P_{e_{4}}=P_{e_{5}}$. Thus, the illegal codeword is the reason for a soft-decision decoder being unable to asymptotically outperform a hard-decision decoder.

In the section, we studied soft-decision decoding. Union bounds are used to investigate the decoder properties. We showed that the illegal codewords are the reason for degrading of decoding performance. 


\section{MaXimum-Likelihood Decoding With Overall NETWORK INFORMATION}

Now we assume the sink knows the statistic information of upstream channels. We will show how ML decoding is performed using those information. The performance and properties of ML decoding will also be studied in this section.

If statistical information on the upstream channels (overall network information) is available to the sink, the decoder will perform better. We call it overall ML decoding or just ML decoding. Now we show how the ML decoder works. We let $\underline{C}_{i, j}(j=1, \cdots, J)$ the final hop codeword when $\underline{I}_{i}(i=0, \cdots, K-1)$ is source information and the error pattern $Z_{j}$ occurs. $\underline{Y}$ is the sink received signal vector. The optimal decoder [19], [11] (maximum $a$-posteriori or MAP decoder) decodes as

$$
\arg \max _{\underline{I}_{i}} P\left(\underline{I}_{i} \mid \underline{Y}\right)=\arg \max _{\underline{I}_{i}} \frac{P\left(\underline{I}_{i}\right) P\left(\underline{Y} \mid \underline{I}_{i}\right)}{P(\underline{Y})} .
$$

If all $\underline{I}_{i} \mathrm{~s}$ have the same probabilities, the decoder is a ML decoder and (5) reduces to ([19], [11])

$$
\begin{aligned}
\arg \max _{\underline{I}_{i}} P\left(\underline{Y} \mid \underline{I}_{i}\right) & =\arg \max _{\underline{I}_{i}} \sum_{\underline{C}_{i, j}} P\left(\underline{C}_{i, j} \mid \underline{I}_{i}\right) P\left(\underline{Y} \mid \underline{C}_{i, j}, \underline{I}_{i}\right) \\
& =\arg \max _{\underline{I}_{i}} \sum_{\underline{C}_{i, j}} P\left(\underline{C}_{i, j} \mid \underline{I}_{i}\right) P\left(\underline{Y} \mid \underline{C}_{i, j}\right) .
\end{aligned}
$$

(6) follows since $\underline{Y}$ is conditionally independent of $\underline{I}_{i}$ if $\underline{C}_{i, j}$ is given. The decoder calculates $\sum_{j=1}^{J} P\left(\underline{C}_{i, j} \mid \underline{I}_{i}\right) P\left(\underline{Y} \mid \underline{C}_{i, j}\right)$ for all $\underline{I}_{i}$ and decodes the one with the maximum value. $P\left(\underline{Y} \mid \underline{C}_{i, j}\right)$ is determined by the final hop channels. $P\left(\underline{C}_{i, j} \mid \underline{I}_{i}\right)=P\left(Z_{j}\right)$. Since the sink knows the error probabilities of the upstream channels, it can calculate all error patterns and their probabilities. The decoder can perform overall ML decoding. The simulation results for the system in Fig. 1 are shown in Fig. 2. We can see that statistical information of upstream channels can significantly improve the BEP. To further disclose properties, we derive the union bound as follows.

We assume $J$ error patterns with the probabilities $P\left(Z_{j}\right)$ $(j=1, \cdots, J)$. Each $\underline{I}_{i}$ has a unique final hop codeword $\underline{C}_{i, j}$ under $Z_{j}$ (since each $\underline{I}_{i}$ has a unique correct final hop codeword $\underline{C}_{c, i}$, and $\underline{C}_{i, j}=\underline{C}_{c, i}+Z_{j}$ ). Assuming that $\underline{I}_{0}=$ $\{0,0, \cdots, 0\}$ is transmitted, the union bound is

$$
P_{b, O} \leq \sum_{j=1}^{J} P\left(Z_{j}\right) \sum_{i=1}^{K-1} P\left(\underline{I}_{i} \mid Z_{j}\right) \frac{w_{i}}{w}
$$

where $P\left(\underline{I}_{i} \mid Z_{j}\right)$ is the probability in which the error pattern $Z_{j}$ occurs, and $\underline{I}_{i}$ is more probable than $\underline{I}_{0} . w_{i}$ is the weight [11] of $\underline{I}_{i}$. Clearly, $P\left(\underline{I}_{i} \mid Z_{j}\right)=P\left(\underline{I}_{i} \mid \underline{C}_{0, j}\right)$. From (6), $P\left(\underline{I}_{i} \mid \underline{C}_{0, j}\right)$ is the probability of the received vector falling into a region in $\mathbb{R}^{N}$ where the following inequality satisfies

$$
\begin{gathered}
\sum_{m=1}^{J} P\left(\underline{C}_{i, m} \mid \underline{I}_{i}\right) P\left(\underline{Y}_{j} \mid \underline{C}_{i, m}\right) \geq \\
\sum_{m=1}^{J} P\left(\underline{C}_{0, m} \mid \underline{I}_{0}\right) P\left(\underline{Y}_{j} \mid \underline{C}_{0, m}\right),
\end{gathered}
$$

where $\underline{Y}_{j}=\underline{X}_{0, j}+\underline{n}$ is the received vector. (8) means that $\underline{I}_{i}$ is more probable than $\underline{I}_{0}$ under ML decoding. $\underline{X}_{0, j}=$ $\pi\left(\underline{C}_{0, j}\right)$. Since $P\left(\underline{C}_{0, j} \mid \underline{I}_{0}\right)=P\left(\underline{C}_{i, j} \mid \underline{I}_{i}\right)=P\left(Z_{j}\right)$ for $j=$ $1, \cdots, J,(8)$ is the same as

$$
\sum_{m=1}^{J} P\left(Z_{m}\right) P\left(\underline{Y}_{j} \mid \underline{C}_{i, m}\right) \geq \sum_{m=1}^{J} P\left(Z_{m}\right) P\left(\underline{Y}_{j} \mid \underline{C}_{0, m}\right) \text {. }
$$

For the example in Fig. 1, the received vectors [19] under the error patterns $Z_{1}, Z_{2}$ are

$$
\begin{aligned}
& Z_{1}: \underline{Y}_{1}=\left\{-\sqrt{E_{b}},-\sqrt{E_{b}},+\sqrt{E_{b}}\right\}+\left\{n_{1}, n_{2}, n_{3}\right\}, \\
& Z_{2}: \underline{Y}_{2}=\left\{-\sqrt{E_{b}},-\sqrt{E_{b}},-\sqrt{E_{b}}\right\}+\left\{n_{1}, n_{2}, n_{3}\right\} .
\end{aligned}
$$

To decode, we need to consider all possible information as $\underline{I}_{0}=\{0,0\}, \underline{I}_{1}=\{0,1\}, \underline{I}_{2}=\{1,0\}$ and $\underline{I}_{3}=\{1,1\}$. Then, $P\left(\underline{I}_{1} \mid Z_{1}\right)$ is the probability of the inequality holding

$$
\begin{aligned}
& P\left(Z_{1}\right) P\left(\underline{Y}_{1} \mid \underline{C}_{1,1}\right)+P\left(Z_{2}\right) P\left(\underline{Y}_{1} \mid \underline{C}_{1,2}\right) \geq \\
& P\left(Z_{1}\right) P\left(\underline{Y}_{1} \mid \underline{C}_{0,1}\right)+P\left(Z_{2}\right) P\left(\underline{Y}_{1} \mid \underline{C}_{0,2}\right),
\end{aligned}
$$

where $\underline{C}_{i, m}=\underline{C}_{i}+Z_{m}$ with $i=0,1$ and $m=1,2$. Here $\underline{C}_{0}$ and $\underline{C}_{1}$ are the correct final-hop codewords for $\underline{I}_{0}$ and $\underline{I}_{1}$, respectively. For BPSK, (11) becomes

$$
\begin{array}{r}
P\left(Z_{1}\right) \exp \left(-\left(8 E_{b}+4 \sqrt{E_{b}}\left(n_{3}-n_{2}\right)\right) / N_{0}\right)+ \\
P\left(Z_{2}\right) \exp \left(-\left(4 E_{b}-4 \sqrt{E_{b}} n_{2}\right) / N_{0}\right) \geq \\
P\left(Z_{1}\right)+P\left(Z_{2}\right) \exp \left(-\left(4 E_{b}+4 \sqrt{E_{b}} n_{3}\right) / N_{0}\right) .
\end{array}
$$

In (12), only $n_{2}$ and $n_{3}$ are random variables (RV). They have a Gaussian distribution, zero-mean and a variance $\sigma^{2}=$ $N_{0} / 2$ [19]. Thus, the probability of this inequality to hold equals the event when $n_{2}$ and $n_{3}$ fall in a region of $\mathbb{R}^{2}$ where (12) holds. Equivalently, it is the probability of following event: The signal $\{0,0\}$ is transmitted, and it is moved out of its decision region by the noise vector $\left\{n_{2}, n_{3}\right\}$. The boundary of the decision region for the signal vector $\{0,0\}$ is where (12) holds with equality. The vector $\{0,0\}$ is called an equivalent point (in $\mathbb{R}^{2}$ ). We should find this boundary, and use (4) to get the event probability $P\left(\underline{I}_{1} \mid Z_{1}\right) \approx Q\left(d_{\min } \sqrt{\frac{2}{N_{0}}}\right)$ for the medium-to-high SNR. Here $d_{\min }^{2}=\min n_{2}^{2}+n_{3}^{2}$ with a constraint of (12). It is the minimum distance from the point $\{0,0\}$ to the boundary. From (12), the boundaries (and $d_{\text {min }}$ ) changes with $E_{b} / N_{0}(\mathrm{SNR})$. The boundaries of various SNRs are shown in Fig. 5. Thus, the points in the boundaries with the minimum distance $d_{\min }$ to $\{0,0\}$ has $n_{3}=0$. Then, $d_{\min }=n_{2}$ with a constraint of the equality of (12) holding. By setting $n_{3}=0$, we can easily get

$$
d_{\text {min }}=n_{2}=\frac{\log \left(\frac{e^{-E_{b}}+e^{-4 E_{b}}}{e^{-9 E_{b}+e^{-4 E_{b}}}}\right)}{4 \sqrt{E_{b}}} \approx \frac{3 \sqrt{E_{b}}}{4} .
$$

Thus, $P\left(\underline{I}_{1} \mid Z_{1}\right) \approx Q\left(\sqrt{\frac{9}{8} \mathrm{SNR}}\right)$ for the medium-to-high SNR. Similarly, we can calculate all $P\left(\underline{I}_{i} \mid Z_{j}\right)$ and finally evaluate (7). The bound and simulations are shown in Fig. 6. The bound and the simulation results are asymptotically offset. The bound shows the asymptotic performance of the decoder.

The computation procedure of $P\left(\underline{I}_{i} \mid Z_{j}\right)$ is summarized as follows. Simplifying (8), we find the boundary where equality in (8) holds in $M$-dimensional Euclidean space. We assume 


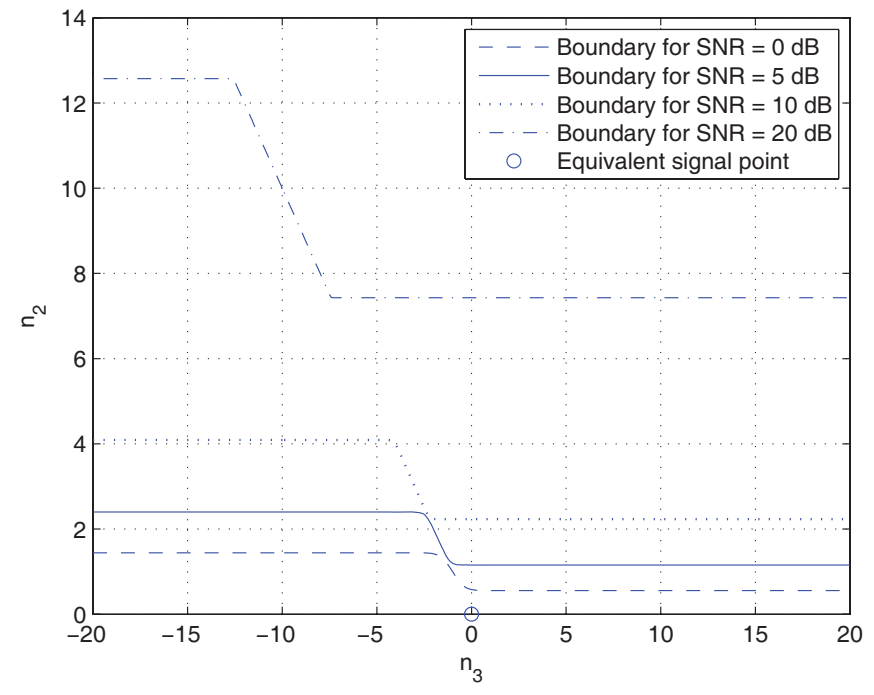

Fig. 5. The boundaries for the decision region of the equivalent point procedure in $\mathbb{R}^{2}$. The equivalent transmitted vector is at the original point, and an error occurs when noise moves the vector out of the boundaries. Boundaries are $\left(n_{2}, n_{3}\right)$ with equality of (12) holding.

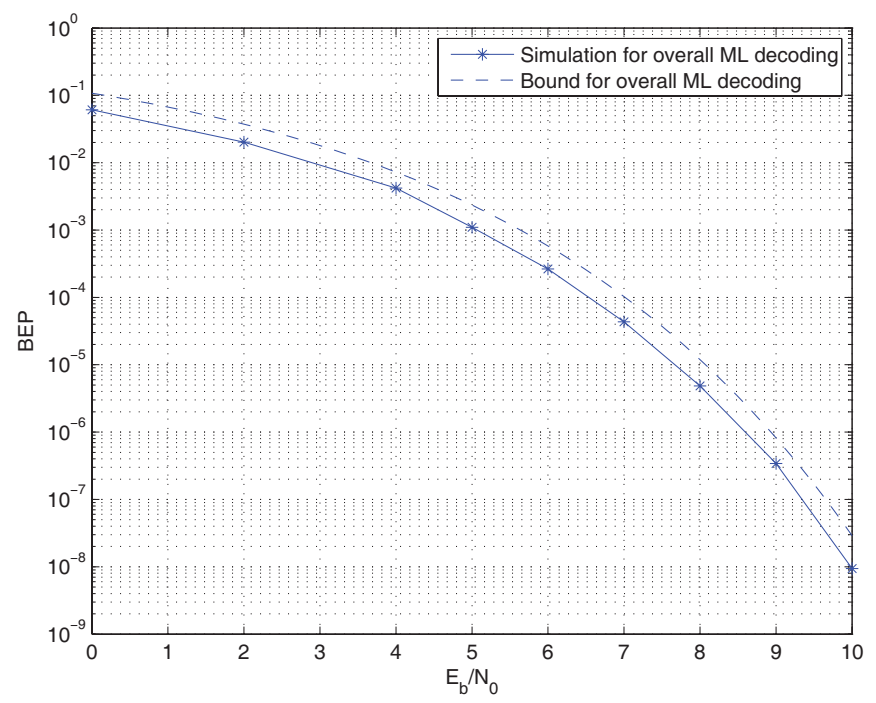

Fig. 6. Simulation results and the union bound of the overall ML decoder for the system in Fig. 1.

$N$ final hop channels. $M \leq N$, since part of the noise variables can be removed in simplification. Thus, $P\left(\underline{I}_{i} \mid Z_{j}\right)$ is the probability in which the transmitted vector at the original point is moved out of the boundary by noise in $\mathbb{R}^{M}$. To evaluate the probability, we find the minimum distance $d_{\min }$ from the boundary to the original point. Finally, we can calculate $P\left(\underline{I}_{i} \mid Z_{j}\right)$ by (4). Since $\{0,0, \cdots\}$ is used as the equivalent transmitted point, we call the procedure as equivalent point procedure. The difficulty is to find $d_{\min }$. We cannot always calculate $d_{\min }$ by manually observing the boundary in the Euclidean signal space. An analytical approach is often needed. Formally, $d_{\min }^{2}$ is the minimum squared value of the sum of squared noise variables with the constraint of equality in (8). Thus, the problem of finding $d_{\min }$ is reduced to the following optimization problem:

Minimize $d^{2}=n_{1}^{2}+n_{2}^{2}+\cdots+n_{M}^{2}$
S. T $\sum_{m=1}^{J} P\left(Z_{m}\right) P\left(\underline{Y}_{j} \mid \underline{C}_{i, m}\right)=\sum_{m=1}^{J} P\left(Z_{m}\right) P\left(\underline{Y}_{j} \mid \underline{C}_{0, m}\right)$.

This is a least-squares problem with a non-convex constraint [28]. In general, it cannot be efficiently solved [28]. Yet, with an increased SNR, one term in the RHS or LHS sum of the constraint will dominate the RHS and LHS, respectively. Thus, the constraint can be greatly simplified. It can become linear for medium-to-high SNR. Thus, an analytical solution of the optimization problem is available [28] and $d_{\min }$ can be found. For example, in (12), the LHS $\approx \exp \left(-\left(4 \mathrm{E}_{b}-4 \sqrt{\mathrm{E}_{b}} n_{2}\right) / N_{0}\right)$. Another term of the LHS is $\exp \left(-\left(9 \mathrm{E}_{b}-4 \sqrt{\mathrm{E}_{b}}\left(n_{3}-n_{2}\right)\right) / N_{0}\right)$ and is quite small for medium-to-high SNR. Thus, it can be ignored to simplify. Note that $n_{i}(i=1, \cdots, M)$ are zero-mean RVs with a Gaussian distribution. Thus the probability of $E_{b}$ being larger than the $n_{i}$ (and their linear combination) approaching 1 with increasing SNR in (11), (12). Then, we only need to consider the coefficient of $\mathrm{E}_{b}$. Here we use the approximation $Q(x) \approx$ $0.5 \exp \left(-0.5 x^{2}\right)$ [19]. Thus, $P\left(Z_{1}\right) \approx \exp \left(-\mathrm{E}_{b} / N_{0}\right)$ and $P\left(Z_{2}\right) \approx 1$. For the same reason, the RHS $\approx \exp \left(-\mathrm{E}_{b} / N_{0}\right)$. This gives $d_{\min } \approx \frac{3 \sqrt{E_{b}}}{4}$, the same as (13). Thus, we can evaluate the bound by solving the optimization problem.

In this section, we studied the overall ML decoder. We showed how to perform ML decoding with error patterns and their probabilities of final-hop codewords. We also showed how to evaluate them from statistical information on the upstream channels. The union bounds are derived to evaluate the asymptotical performance of the ML decoder.

\section{Genie-Aided Decoder And Genie Bound}

In this section, we will investigate the performance and properties of the decoders, which know deterministically the error patterns of every received packet.

\section{A. Genie-Aided Decoder}

We assume the sink knows the error pattern of each finalhop codeword from a genie [29]. The decoder is therefore called the genie decoder. The main motivation for us to investigate the genie decoder is to get a BEP performance lower bound, since the genie-aided decoding should outperform these without a genie aid [29]. Also, as we will show, the performance bound for the genie decoder is quite straightforward to evaluate, and it is tight for practical BEP regions (below $10^{-2}$ ). First, we show how the decoder works.

In overall ML decoding, the decoder does not know the error pattern of one specific received codeword. Thus, one source information vector $\underline{I}_{i}$ can lead to multiple final hop codewords $\underline{C}_{i, j}$ in probabilities. The mapping from $\underline{I}_{i}$ to $\underline{C}_{i, j}$ is one-to-many. The process is shown in the LHS of Fig. 7. The overall ML decoder needs to consider all possible error patterns for one $\underline{I}_{i}$. In the figure, there are $K \underline{I}_{i}$ s, and $J$ error patterns. The probability of the $i$ th error pattern is $P\left(Z_{i}\right)$. With the genie aid, the decoder knows deterministically which error pattern occurs for each received $\underline{Y}$. Thus, $\underline{I}_{i}$ and $\underline{C}_{i, j}$ is a one-to-one mapping. The process is shown in the RHS of Fig. 7. Since the genie tells the error pattern, the decoder only calculates which $\underline{C}_{i, j}$ is the most probable. The genie decoder decides $\underline{I}_{i}$ by

$$
\arg \max _{\underline{I}_{i}} P\left(\underline{Y} \mid \underline{C}_{i, j}\right)
$$




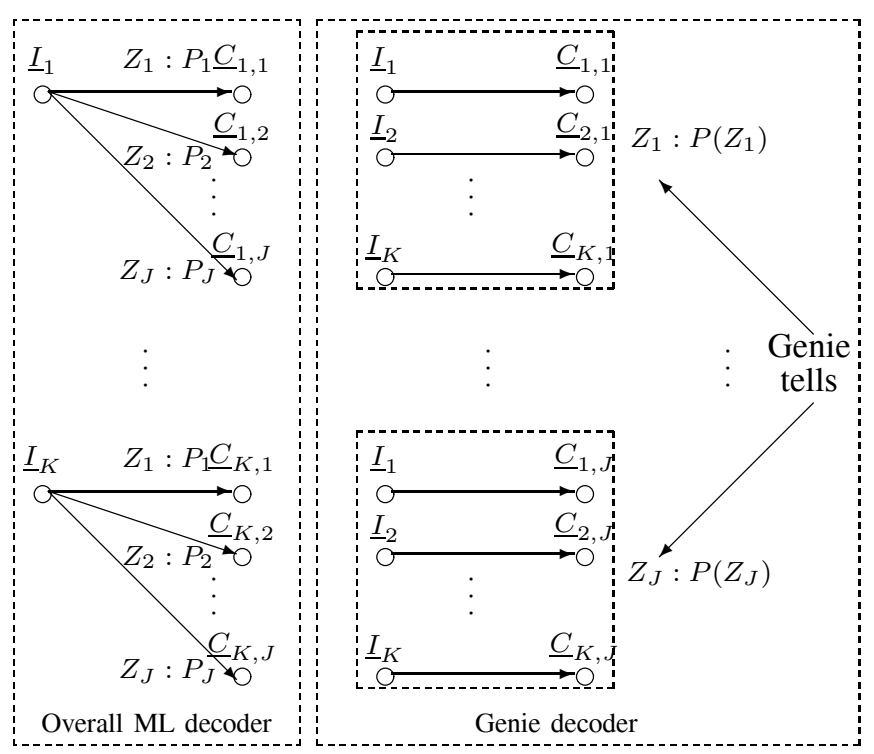

Fig. 7. Comparison of overall ML decoding and genie-aided decoding. $\underline{C}_{i, j}$ is the final hop codeword caused by the information vector $\underline{I}_{i}$ and the error pattern $Z_{j} . P\left(Z_{j}\right)$ is the probability of $Z_{j}$.

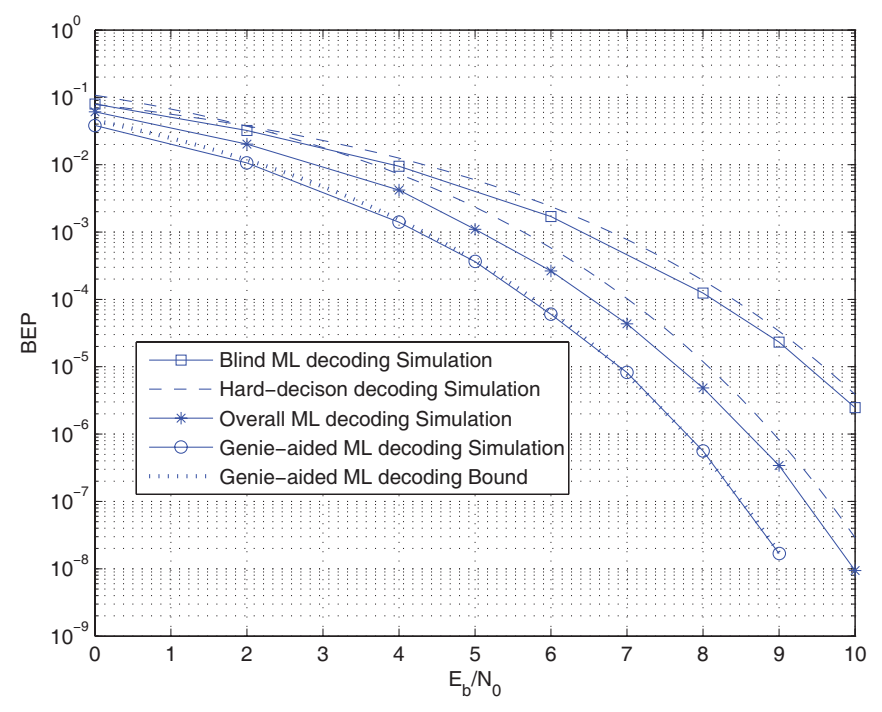

Fig. 8. Comparison of simulation results for hard-decision decoding, softdecision decoding, overall ML decoding and Genie-aided decoding, and their bounds.

The simulations of the genie decoder for the system in Fig. 1 are shown in Fig. 8. For comparison, we also show the numerical results for the overall ML decoder in Fig. 8. We can see that the genie decoder has better performance.

\section{B. Genie Bound}

To analyze the performance of the genie decoder, we use the union bound. Assuming the all-zero vector $\underline{I}_{0}$ as source information, the union bound of the genie decoder is

$$
P_{b, G} \leq \sum_{m=1}^{J} P\left(Z_{m}\right) \sum_{n=1}^{K} P\left(\underline{C}_{n, m} \mid \underline{C}_{0, m}\right) \frac{w_{n}}{w},
$$

where $P\left(\underline{C}_{n, m} \mid \underline{C}_{0, m}\right)$ is the probability of $\underline{C}_{n, m}$ (thus $\underline{I}_{n}$ ) being decoded, and $w_{n}$ is the weight of $\underline{I}_{n}$. We can see that the genie bound is much easier to compute than for the ML decoder. The numerical results are shown in Fig. 8, the bound and simulations closely match in practical BEP regions.

In the section, we investigated the genie-aided decoder. With more knowledge of upstream channels, the decoder has better performance (than the ML decoder). As a performance lower bound (for various decoders), the genie bound is quite straightforward to calculate.

\section{CONCLUSIONS AND DISCUSSION}

We investigated hard-decision decoding, soft-decision decoding, ML decoding and genie-aided decoding approaches for coded networks with noisy channels. We showed how these decoding approaches should be performed with different knowledge of upstream channels, and presented analysis bounds for them. For these decoding approaches, more and more information on the upstream channels is used resulting in improved performance. Thus, we can conclude that knowledge of upstream channels is essential to the sink performance.

Above, we assume no direct channels between different sinks. If there are channels between them, and further these channels are error-free, the sinks can freely exchange decoding information. Thus, we can logically regard these sinks as one virtual sink in the decoding sense. Then, the sinks can use the channel observations from all channels input to them. The decoding approach is the similar to the single sink case. Yet, we note that error-free connections between sinks are very ideal in practice.

For the networks with interference channels, analysis is more involved. Yet similar decoding approaches may be developed for such networks: First, we collect the error patterns for the final hop codewords, and then use equations similar to (5)-(6) to perform ML decoding. For this, we need to modify the algorithm of collecting error patterns. For networks with interference channels, it is hard to enumerate all ME error events. Then, we can turn to approximate results on error patterns by only considering dominant error events of the upstream channels. Also, new equations should be developed for ML decoding with interference channels, which are similar to the ML decoding for one-hop interference channels (see, for instance, [11], [30]), since there is only one hop from the final hop set to the sinks. These can be directions for future work. Here we do not go further, due to space limitations.

\section{APPENDIX}

\section{A. Error Pattern Collection}

To evaluate the error patterns, we use the transfer matrix defined in [3]. It denotes the relation of source information bits and the sink reproduced bits. The transfer matrix is calculated as [3]

$$
\underline{M}=\underline{A} \cdot \underline{P} \cdot \underline{B},
$$

where $\underline{A}$ is the coding matrix between the source information bits and the input to the edges connected to the source nodes (We call it the network input for convenience.), $\underline{B}$ is the transfer matrix between the sink received bit and reproduced bits, and $\underline{P}$ is the transfer matrix between the network input and the sink received bits. We call the matrix $\underline{H}=\underline{A} \cdot \underline{P}$ 
the encoding matrix. The reproduced bits at the sinks are calculated as $\underline{Z}=\underline{I} \cdot \underline{M}$, where $\underline{Z}$ are sink reproduced bits (row vector). Thus, a row in $\underline{M}$ corresponds to a source bit and a column to a sink reproduced bit. A nonzero element (say $i$-th row and $j$-th column) in $\underline{M}$ means the $i$-th source bit is transmitted to the $j$-th reproduced bit. Thus, there is a logical path between the $i$-th source bit and $j$-th reproduced bit. For Fig. $1, \underline{H}$ is

$$
\underline{H}=\left(\begin{array}{ccc}
\alpha_{1, e_{6}} \beta_{e_{6}, e_{1}} & \alpha_{1, e_{6}} \beta_{e_{6}, e_{4}} \beta_{e_{4}, e_{3}} & 0 \\
0 & \alpha_{2, e_{7}} \beta_{e_{7}, e_{5}} \beta_{e_{5}, e_{3}} & \alpha_{2, e_{7}} \beta_{e_{7}, e_{2}}
\end{array}\right) .
$$

The indeterminate variables $\alpha_{i, e_{j}}$ and $\beta_{e_{i}, e_{j}}$ are the coding variables. Note that the second subscripts of $\alpha_{i, e_{j}}$ and $\beta_{e_{i}, e_{j}}$ denote the channel labels for their encoding output. This $2 \times 3$ matrix denotes the relation between two source bits and three sink received bits. A more detailed description of the transfer matrix is given in [3]. To evaluate the error patterns, we first consider the states of every channel in $\bar{E}(t)$. Here the state shows if the channel has a transmission error or not. Each channel is in one of two states: Having an error or having no error. An error event is redefined as one or more channel in $\bar{E}(t)$ having errors. Thus, we can collect all error events. Since the channels are independent, the error events are ME. For an error event, a dummy variable is used to trace how channel errors propagate and cancel in the network. It is defined as follows:

Definition 5. T: A symbolic variable is used to denote a channel error in $\bar{E}(t)$. It follows the rules:

$$
T \cdot T=1
$$

and

$$
T+T=0 .
$$

(18) tells that two channel errors in the same path will cancel the transmitted error since the transmitted symbols are binary. The second transmitted error will change the error bit back to the correct one. (19) tells that two bit errors will cancel when they are added. Then, we calculate $\underline{H}$ for the error event. Note that each column in $\underline{H}$ corresponds to a final hop channel. Thus, it corresponds to one bit of the final hop codeword and one bit of the error pattern. For an error event, if a bit of the pattern is 1 or 0 depends on the value of the dummy variable $T$ : An odd number of $T$ s denotes 1 and an even number means 0 . We can determine each bit of the pattern. The probability of the pattern is the probability of the error event, and is evaluated as

$$
P(c)=\prod_{e_{i} \in E(c)} P_{e_{i}} \cdot \prod_{e_{i} \in \bar{E}(t) \backslash E(c)}\left(1-P_{e_{i}}\right),
$$

where $P_{e_{i}}(i=1, \cdots,|\bar{E}(t)|)$ is the BEP of channel $e_{i}$, and $E(c) \subseteq \bar{E}(t)$ is the error channel set of the event $c$. Different error events may cause the same error pattern. The probability of the pattern is the sum of these error events. Since the error patterns are caused by ME upstream error events, they are ME, and their probabilities add to 1 . The calculation is shown in Algorithm 7.1.

In Fig. 1, $\underline{C}(t)$ (for channels $e_{1}, e_{2}$ and $e_{3}$ ) is $\left\{c_{1}, c_{2}, c_{3}\right\}=$ $\left\{b_{1}, b_{2}, b_{1} \oplus b_{2}\right\}$, if the upstream channels are error-free. Since $e_{6}$ and $e_{7}$ are error-free, $\bar{E}(t)=\left\{e_{4}, e_{5}\right\}$. Assuming all-zero
Algorithm 7.1: (The error pattern and error probability collecting algorithm.)

1: Initialization. Set the error pattern set as empty. Form the matrices $\underline{A}$ and $\underline{P}$ for the coding scheme. All nonzero elements are temporarily kept as indeterminate variables.

2: Calculate the encoding matrix $\underline{H}=\underline{A} \cdot \underline{P}$

3: Mark channel. Replace the indeterminate variables $\left(\alpha_{i, e_{j}}\right.$ and $\left.\beta_{e_{i}, e_{j}}\right)$ in $\underline{H}$ with its second subscript, which denotes a channel. If the channel is a final hop channel $E(t)$, remove its symbol.

4: Remove redundant channel symbols: For each column of the matrices $\underline{H}$, if there are more than one symbols for any channel, only keep one symbol for it and remove all other symbols for this channel by setting them to 1 .

5: Mark error. Enumerating all error events in $\bar{E}(t)$. For each column of the matrix $\underline{H}$, if any channel is assumed to be erroneous in the error event, replace its symbol with $T$. Set all symbols for the correct channels to $1 \mathrm{~s}$.

6: Calculation of $\underline{H}$. Simplify $\underline{H}$ by using equations (18) and (19).

7: Make decision for each bit of the error pattern. For each column of $\underline{H}$, if there are a total of odd $T \mathrm{~s}$, then the bit corresponding to this column is 1 , otherwise, it is 0 .

8: Calculate the probability of the error event using (20).

9: If the error pattern already exist in the error pattern set, just add the error probability (with the probability of the existed pattern). Otherwise, insert the error pattern and the error probability in the set. 10: Collect all error patterns and error probability, and output results.

information, the correct final hop codeword is $\underline{C}_{c}=\{0,0,0\}$. We collect error patterns by enumerating the states of all channels of $\bar{E}(t)$ using Algorithm 7.1. With $\underline{H}$ in (17), we mark channels and get $\underline{H}=\left(\begin{array}{ccc}1 & e_{4} & 0 \\ 0 & e_{5} & 1\end{array}\right)$. For $\underline{H}$, we only consider the channel set $\bar{E}(t)=\left\{e_{4}, e_{5}\right\}$ and remove other channel marks. For the error event when $e_{4}$ has an error and $e_{5}$ has no error, $\underline{H}$ is marked as $\left(\begin{array}{ccc}1 & T & 0 \\ 0 & 1 & 1\end{array}\right)$. The second column has one $T$. Thus, the output from $m_{3}$ has an error. The error pattern is $Z_{1}=\{0,0,1\}$ for the error event. The probability of the error pattern is the probability of the error event $P=\left(1-P_{e_{5}}\right) P_{e_{4}}$. Similarly, we can obtain all error patterns and their probabilities as

$$
\begin{aligned}
Z_{1}=\{0,0,1\}: P\left(Z_{1}\right) & =\left(1-P_{e_{5}}\right) P_{e_{4}}+\left(1-P_{e_{4}}\right) P_{e_{5}} \\
& =2 P_{e}-2 P_{e}^{2} \\
Z_{2}=\{0,0,0\}: P\left(Z_{2}\right) & =P_{e_{5}} P_{e_{4}}+\left(1-P_{e_{4}}\right)\left(1-P_{e_{5}}\right) \\
& =1-2 P_{e}+2 P_{e}^{2} .
\end{aligned}
$$

Adding $\{0,0,0\}$, the final hop codewords and their probabilities are

$$
\begin{array}{r}
\underline{C}_{1}=\{0,0,1\}: P\left(\underline{C}_{1}\right)=P\left(Z_{1}\right) ; \mathrm{AND} \\
\underline{C}_{2}=\{0,0,0\}: P\left(\underline{C}_{2}\right)=P\left(Z_{2}\right) .
\end{array}
$$

\section{B. Bound Analysis for Soft-Decision Decoding of Fig. 1}

For soft-decision decoding, the candidate codewords are: $\widetilde{\widetilde{C}_{1}}=\{0,0,0\},{\widetilde{C_{2}}}_{2}=\{0,1,1\},{\widetilde{C_{3}}}_{3}=\{1,0,1\}$ and $\widetilde{C}_{4}=$ $\{1,1,0\}$. They are for information vectors $\underline{I}_{1}=\{0,0\}$, $\underline{I}_{2}=\{0,1\}, \underline{I}_{3}=\{1,0\}$ and $\underline{I}_{4}=\{1,1\}$, respectively. The illegal final hop codeword $\underline{C}_{1}=\{0,0,1\}$ is not a candidate codeword. The transmitted vectors (points in $\mathbb{R}^{3}$ ) of candidate codewords and final hop codewords are shown in Fig. 9. In the figure, $\pi\left(\underline{C}_{1}\right)$ has the same distances to $\pi\left(\underline{\widetilde{C}}_{1}\right), \pi\left(\widetilde{\widetilde{C}}_{2}\right)$ and $\pi\left(\widetilde{\widetilde{C}}_{3}\right)$. Among them, $\pi\left({\widetilde{C_{1}}}_{1}\right)$ is the correct one. $\pi\left(\underline{C}_{1}\right)$ is exactly at the border of the decision region. 


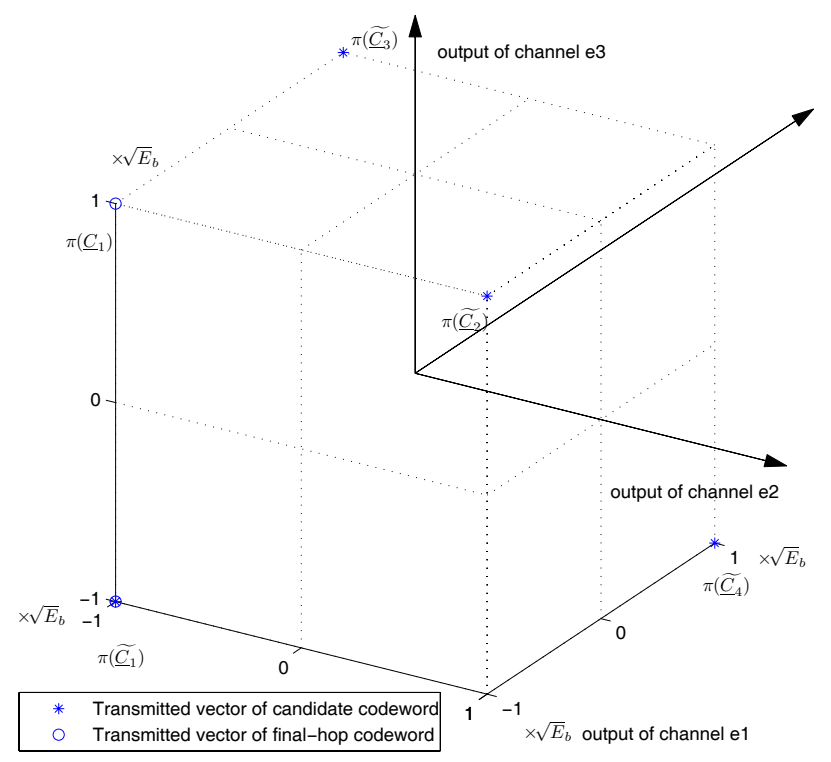

Fig. 9. Geometric representation of the transmitted and received vectors of Fig. 1 in $\mathbb{R}^{3}$. Soft-decision decoding is used in the sinks. BPSK modulation is used for the channels $\left\{e_{1}, e_{2}, e_{3}\right\}$.

The decision region $\left(R_{k}\right)$ for $\widetilde{C_{k}}$ is the region in $\mathbb{R}^{3}$ that the receiver decides for $\widetilde{C}_{k}$ [19], [11]. If $\underline{C}_{1}$ is transmitted, very little noise along the wrong codeword $\left(\underline{\widetilde{C}}_{2}\right.$ or $\left.\widetilde{C}_{3}\right)$ direction can move $\pi\left(\underline{C}_{1}\right)$ into wrong decision regions. In Fig. 9, $\pi\left(\underline{C}_{1}\right)$ has equal distances to $\pi\left(\widetilde{C_{1}}\right), \pi\left(\widetilde{C_{2}}\right)$ and $\pi\left(\widetilde{C_{3}}\right) \cdot \widetilde{C_{1}}$ is the correct final hop codeword $\underline{C}_{c}$. Thus, it lies exactly on the border of the decision regions. Thus, $\hat{d}_{1,2}=0$ and $P\left(\widetilde{C}_{c} \rightarrow{\widetilde{C_{1}}}_{2} \mid \underline{C}_{1}\right)=0.5$ by (4). In the same way, we can calculate $P\left(\underline{C}_{c} \rightarrow \underline{C}_{3} \mid \underline{C}_{1}\right)=0.5$. When only $\underline{C}_{4}$ and $\widetilde{C}_{c}$ are considered, the border for the decision regions is a plane between them. The distance between $\pi\left(\underline{C}_{1}\right)$ and the plane is $\sqrt{2 E_{b}} . P\left(\widetilde{\widetilde{C}}_{c} \rightarrow{\widetilde{C_{4}}}_{4} \mid \underline{C}_{1}\right)=Q\left(\sqrt{\frac{4 E_{b}}{N_{0}}}\right)$. Through similar calculation, we can get the union bound as

$$
\begin{aligned}
P_{b, s} \leq P\left(\underline{C}_{1}\right)\left(0.5 \frac{1}{w}\right. & \left.+0.5 \frac{1}{w}+\frac{2}{w} Q\left(\sqrt{\frac{4 E_{b}}{N_{0}}}\right)\right) \\
+ & P\left(\underline{C}_{2}\right)\left(2 Q\left(\sqrt{\frac{4 E_{b}}{N_{0}}}\right)\right),
\end{aligned}
$$

where $w=2$ is the number of the information bits. The simulation and bound are shown in Fig. 4. The union bound is offset. Thus, the bound can describe the asymptotic behavior for soft-decision decoding. The bound is not asymptotically tight because the decision regions $\hat{R}_{2}$ and $\hat{R}_{3}$ are overlapping when pair-wise probabilities are considered. Meanwhile, $P\left(\underline{\widetilde{C}}_{c} \rightarrow \widetilde{C}_{2} \mid \underline{C}_{1}\right)$ and $P\left(\widetilde{\widetilde{C}}_{c} \rightarrow \widetilde{C}_{3} \mid \underline{C}_{1}\right)$ are positive. Thus, $P\left(\underline{C}_{2} \mid \underline{C}_{1}\right)+P\left(\underline{C}_{3} \mid \underline{C}_{1}\right)<P\left(\underline{\widetilde{C}}_{c} \rightarrow \underline{C}_{2} \mid \underline{C}_{1}\right)+$ $P\left(\underline{\widetilde{C}}_{c} \rightarrow \widetilde{C}_{3} \mid \underline{C}_{1}\right)$. In (23), 0.5 $\frac{1}{w}$ is constant to the SNR. $P\left(\underline{C}_{2}\right)\left(2 Q\left(\sqrt{\frac{4 E_{b}}{N_{0}}}\right)\right) \ll P\left(\underline{C}_{1}\right)$ for the medium-to-high SNR. Thus, the union bound is asymptotically dominated by $P\left(\underline{C}_{1}\right)$. The bound curve is up-shifted (from the exact values).

\section{REFERENCES}

[1] R. Ahlswede, N. Cai, S.-Y. R. Li, and R. W. Yeung, "Network information flow," IEEE Trans. Inform. Theory, pp. 1204-1216, July 2000.

[2] S. Li, R. W. Yeung, and N. Cai, "Linear network coding," IEEE Trans. Inform. Theory, vol. 49, pp. 371-381, Feb. 2003.

[3] R. Koetter and M. Medard, "An algebraic approach to network coding," IEEE/ACM Trans. Networking, vol. 11, pp. 782-795, Oct. 2003.

[4] L. Song, N. Cai, and R. W. Yeung, "A separation theorem for single source network coding," IEEE Trans. Inform. Theory, vol. 52, pp. 18611871, May 2006.

[5] C. E. Shannon, "A mathematical theory of communication," Bell System Techn. J., pp. 379-423, 623-656, 1948.

[6] C. E. Shannon, "Coding theorems for a discrete source with a fidelity criterion," in IRE Nat. Conv. Rec., vol. 7, pp. 142-163, Mar. 1959.

[7] E. Ayanoglu and R. M. Gray, "The design of joint source and channel trellis waveform coders," IEEE Trans. Inform. Theory, vol. IT-33, pp. 855-865, Nov. 1987.

[8] J. G. Dunham and R. M. Gray, "Joint source and channel trellis encoding," IEEE Trans. Inform. Theory, vol. IT-27, pp. 516-519, July 1981.

[9] N. Cai and R. W. Yeung, "Network coding and error correction," in Proc. IEEE Info. Theory Workshop, 2002.

[10] S. Lin and D. Costello, Error Control Coding. Pearson Prentice Hall, 2004.

[11] J. Proakis, Digital Communications, 4th ed. McGraw-Hill Press, 2000.

[12] D. Tuninetti and C. Fragouli, "Processing along the way: forwarding vs. coding," in Proc. ISITA2004, Italy, Oct. 2004.

[13] D. Tuninetti and C. Fragouli, "On the throughput improvement due to limited complexity processing at relay nodes," in Proc. IEEE Int. Sym. on Infor. Theory, Sept. 2005.

[14] N. Ratnakar and G. Kramer, "On the seperation of channel and network coding in Aref network," in Proc. IEEE Int. Sym. on Info. Theory, Sept. 2005.

[15] K. Chi and X. Wang, "Analysis of network error correction based on network coding," IEE Proc. Commun., Aug. 2005.

[16] D. S. Lun, M. Médard, R. Koetter, and M. Effros, "Further results on coding for reliable communication over packet networks," in Proc. IEEE Int. Sym. on Info. Theory, Sept. 2005.

[17] J. Sundararajan and H. Viswanathan, "Comparison of schemes for streaming multicast in cellular network with relays," in Proc. IEEE Wireless Commun. and Net. Conf. (WCNC), Las Vegas, USA, Apr. 2006.

[18] http://www.networkcoding.info.

[19] J. M. Wozencraft and I. M. Jacobs, Principles of Communication Engineering. New York: Johan Wiley \& Sons, 1965.

[20] G. D. Forney, Jr. and A. Vardy, "Generalized minimum-distance decoding of Euclidean-space codes and lattices," IEEE Trans. Inform. Theory, vol. 42, pp. 1992-2026, Nov. 1996.

[21] M. Xiao and T. Aulin, "A physical layer aspect of network coding with statistically independent noisy channels," in Proc. IEEE Int. Conf. on Commun., Istanbul, Turkey, June 2006.

[22] M. Xiao and T. Aulin, "Energy-efficient network coding for the noisy channel network," in Proc. IEEE Int. Sym. on Info. Theory, Seattle, USA, July 2006.

[23] O. K. Tonguz and G. Ferrari, Ad Hoc Wireless Networks: A Communication-Theoretic Perspective. Chichester, UK: John Wiley \& Sons, 2006.

[24] A. Scaglione and Y. Hong, "Opportunistic large arrays: cooperative transmission in wireless multihop ad hoc networks to reach far distances," IEEE Trans. Signal Processing, vol. 51, no. 8, pp. 2082-2092, Aug. 2003.

[25] M. Chiang, "Balancing transport and physical layers in wireless multihop networks: jointly optimal congestion control and power control," IEEE J. Select. Areas Commun., Jan. 2005. pp. 104-116.

[26] Y. Wu, P. A. Chou, and S.-Y. Kung, "Minimum-energy multicast in mobile ad hoc networks using network coding," in Proc. IEEE Infor. Theory Workshop, Oct. 2004.

[27] Y. Wu, P. A. Chou, Q. Zhang, K. Jain, W. Zhu, and S.-Y. Kung, "Network planning in wireless ad hoc networks: a cross-layer approach," IEEE J. Select. Areas Commun., Jan. 2005.

[28] S. P. Boyd and L. Vandenberghe, Convex Optimization. Cambridge University Press, 2003.

[29] G. D. Forney, Jr., "Lower bounds on error probability in the presence of large intersymbol interference," IEEE Trans. Commun., pp. 76-77, Feb. 1972.

[30] D. Tse and P. Viswanath Fundamentals of Wireless Communication. Cambridge University Press, 2005. 


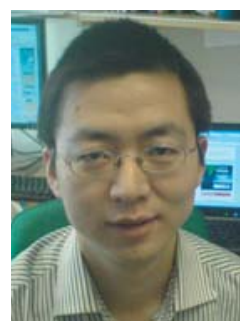

Ming Xiao (S'02-M'07) was born in the SiChuan Province, P. R. China, on May 22nd, 1975. He received Bachelor and Master degrees in Engineering from the University of Electronic Science and Technology of China, ChengDu in 1997 and 2002, respectively. He received Ph.D degree from Chalmers University of technology, Sweden in November 2007. From 1997 to 1999 , he worked as a network and software assistant engineer in ChinaTelecom. From 2000 to 2002, he also held a position in the SiChuan communications administration. From November 2007 to now, he holds a research associate position in ACCESS Linnaeus center, school of electrical engineering, Royal Institute of Technology, Sweden.

The research interest of Dr. Xiao includes: Network Coding (physical layer coding, low-complexity codes, codes for block erasure channels), Rateless Codes (binary deterministic rateless codes with an MDS property), Channel Codes (Turbo codes, LDGM codes), Digital Wireless Communications (Continuous Phase Modulation, Adaptive modulation/codes for fading channels, Cooperative Communications), etc. Dr. Xiao received "Chinese Government Award for Outstanding Self-Financed Students Studying Aborad" in March, 2007. He got "Hans Werthén Grant" from royal Swedish academy of engineering science (IVA) in March 2006. He was a visiting researcher at laboratory for information and decision system, Massachusetts Institute of Technology, USA from Oct. 2006 to Mar. 2007.

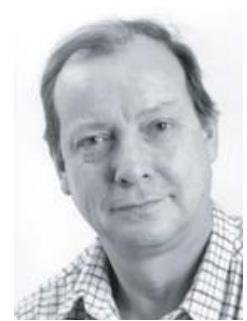

Tor Aulin (S'77-M'80-SM'83-F'99) was born in Malmö, Sweden, on September 12, 1948. He received the M.S. degree in electrical engineering from the University of Lund, Lund, Sweden, in 1974 and the Dr. Techn. (Ph.D.) degree from the Institute of Telecommunication Theory, University of Lund, in November 1979 .

He became a Docent at the University of Lund in 1981 and worked at this institute as a Postdoctoral Fellow. During this period he was also a Visiting Scientist at the ECSE Department at Rensselaer Polytechnic Institute, Troy, NY. Following this he spent one year at the European Space Agency (ESA), the European Space Research and Technology Centre (ESTEC) in Noordwijk, the Netherlands, as an ESA Research Fellow. In 1983 he became a Research Professor (Docent) in Information Theory at Chalmers University of Technology, Göteborg, Sweden. In 1991 he formed the Telecommunication Theory Group there and also became a Docent in Computer Engineering in 1995. During the fall of 1995 he was a Visiting Fellow at the Telecommunications Engineering Department, Australian National University, Canberra, ACT, Australia. He was a Visiting Professor at City University of Hong Kong in 2004 and in 2005 he was a Research scholar at the University of Southern California (USC) in Los Angeles, CA, USA. During 2005 he also spent several months working at Communication Systems Department at Lund University, Lund, Sweden. Some of his research interests are communication theory, combined modulation/coding strategies (such as CPM and TCM), analysis of general sequence detection strategies, digital radio channel characterization, digital satellite communication systems, and information theory. During recent years the potentials of these have been considered for iterative decoding in concatenated versions. This is also the case for such schemes integrated into Multiple Access strategies (TCMA, Trellis Code Multiple Access and its CPM counterpart). Joint source/channel coding also falls into this concept. His company, AUCOM, has performed several advanced theoretical studies as a consultant to some of the major international organizations dealing with developing and operating satellite communication systems, e.g., INTELSAT and ESA. He has also performed theoretical study contracts for Saab and Volvo. Nokia has trusted him as an Internal Lecturer and he has performed numerous studies for Ericsson in the area of digital radio transmission, the latter resulting in a patent. He has authored and published some 200 technical papers and has also authored the book Digital Phase Modulation (Plenum, 1986) as a result of his extensive research in this area at that time. He has organized and chaired several sessions at international symposia/conferences organized by, e.g., IEEE and is an EAMEC representative within the Communications Society of the IEEE. He has been an Editor for IEEE TRANSACTIONS ON COMMUNICATIONS in the area of communication theory and coding for a decade. He is also (for 30 years) on the Communication Theory Committee within IEEE COMSOC.

In December 1997 Dr. Aulin was awarded the Senior Individual Grant at a ceremony in Stockholm, Sweden, handed over by the Prime Minister of Sweden. This has thereafter been repeated in 2004. Dr. Aulin has two papers among the best (Best-of-the-Best) published during the first 50 years of the IEEE COMSOC, selected in connection with their 50th anniversary in 2002. Dr. Aulin also has an academic degree as a solo cellist. 